\title{
Nickel Hydroxide
}

National Cancer Institute

\section{Source}

National Cancer Institute. Nickel Hydroxide. NCI Thesaurus. Code C45863.

A green, crystalline, inorganic compound that produces toxic gases upon heating. Nickel hydroxide is used in nickel-cadmium batteries and as a catalyst in chemical reactions. Exposure to this substance can cause severe dermatitis, skin and asthma-like allergies and affects the lungs, kidneys, gastrointestinal tract and neurological system. Nickel hydroxide is a known carcinogen and is associated with an increased risk of developing lung and nasal cancers. ( $\mathrm{NCl05)}$ 